\title{
C-MRS International Meeting Scheduled for 1990
}

The first "MRS-style" meeting in China will be held in Beijing, May 11-12, 1990. Featuring 14 symposia, the meeting is sponsored by the China Association for Science and Technology, National Science Foundation of China, and the Chinese Academy of Sciences, and is co-sponsored by MRS and E-MRS in addition to 30 other professional societies from the United States, Japan, and China.

Abstract deadline is December 31, 1989. The preregistration fee is $\$ 300$ and will be accepted until December 31. Registration is $\$ 320$ and will be accepted through April 30, 1990.

The meeting will be held at the Jingfeng Hotel in a suburb of Beijing. Abstracts should be sent to and additional information requested from Prof. Heng De $\mathrm{Li}$, Secretary General, C-MRS International '90, c/o Department of Materials Science \& Engineering, Tsinghua University, Beijing 100084, China; telephone 655646,655523 , 666428 , or 2562657; telex 222434 NSFCCN; fax (01) 2019583; cable 6199 Beijing.

Following is a description of the program and topics to be covered.

\section{Symposium A: High T。 Superconductors}

The symposium will emphasize the relationship between superconducting properties and the materials parameters, e.g., preparation of bulk samples, single crystals and film, characterization in structure, chemistry, and physics. Additional topics beyond the above general guidelines will be determined by the latest developments in this rapidly changing field.

Chairs: Zizhao Gan (Peking University, Beijing) and Zhongxian Zhao (Institute of Physics, Academia Sinica, Beijing).

\section{Symposium B: Advanced Composite Materials}

Novel composite materials will be the focus in this symposium, with particular emphasis on:

Novel ideas, methods, and techniques in processing;

- Qualitative, quantitative relationship between properties and structures;

- Physical and chemical compatibilities of material interfaces;

- Characterization of properties for both products and raw materials;

- Suggestion or introduction of modern theories into this area, such as fractal, synergetics, nonlinear mathematics, selfhealing and bionics, etc.

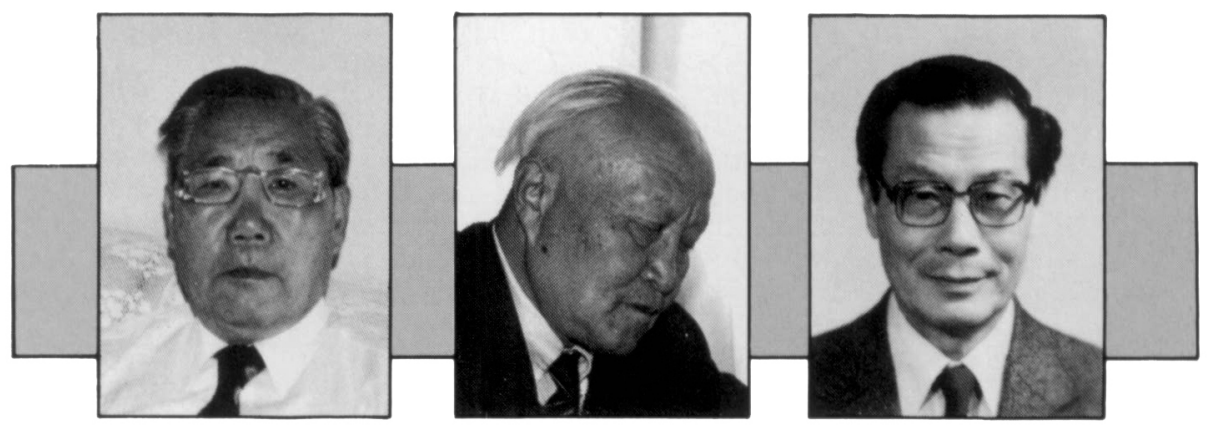

Organizers of the C-MRS meeting (left to right): Heng De Li, Secretary Geneml; Changxu Shi, Conference Chairman and Chairman of the Program Committee; and Dongsheng Yan, Conference Chairman and Chaimnan of the Organization Committee.

Chairs: Renjie Wu (Shanghai Jiao Tong University, Shanghai); E. Fitzer (Karlsruhe University, Karlsruhe); Benlian Zhou (Institute of Metal Research, Academia Sinica, Shenyang); and Chenggong Li (Institute of Aeronautical Materials, Beijing).

\section{Symposium C: High Performance Ceramics}

Ceramic materials for high performance applications have made great strides in recent years for both structural and functional applications. Emphasis in this symposium will be on but not restricted to: Processing science and technology related to fabrication of high performance ceramics;

- Compositional tailoring and microstructural control (submicron to nanometer scale) for high performance ceramics;

- Mechanical properties;

- Ferroelectric, piezoelectric, electro-optic, acousto-optic and other functional properties;

- Applications of high performance ceramics.

Chairs: Jingkun Guo (Shanghai Institute of Ceramics, Academia Sinica, Shanghai); L. Eric Cross (Pennsylvania State University, PA); R.J. Brook (MPI für Metallforschung, Powder Metall. Lab., Stuttgart); Xi Yao (Xian Jiao Tong University, Xian); and Qiming Yuan (Tian Jin University, Tianjin).

\section{Symposium D: Optoelectronic Materials and Functional Crystals}

This symposium will focus on: - Optoelectronic switching, modulating, scanning and processing materials;

- Low dimensional (quantum wells, mi- crocrystallites, etc.) optoelectronic materials;

- Detector and sensor materials;

- Laser materials;

- Nonlinear optical crystals;

- Acoustic and acousto-optical crystal;

- Magneto-optic and electro-optic crystal;

- Piezo- and pyro-electric functional materials;

- Optical storage materials;

- Special optical fibers and fiber sensor materials.

Chairs: Fuxi Gan (Shanghai Institute of Optics and Fine Mechanics. Academia Sinica, Shanghai); R.A. Laudise (AT\&T Bell Laboratories, Murray Hill, NJ); Minhua Jiang (Shandong University, Jinan); Meiying Kong (Institute of Semiconductors, Academia Sinica, Beijing).

\section{Symposium E: Advanced Engineering Plastics}

This symposium will address:

- High performance engineering plastics;

- Thermotropic liquid crystalline polymers;

- Polymer alloys;

- Filler-reinforced polymer composites;

- Deformation, yielding, fracture and toughening mechanism;

- Application of engineering plastics as economical and versatile metal and wood substitutes.

Chairs: $\mathrm{Xi}$ Xu (Chengdu University of Science and Technology, Chengdu); R.S. Porter (University of Massachusetts, Amherst, MA); L.A. Utracki (Industrial Materials Research Institute, NRC, Canada); Zongneng Qi (Institute of Chemistry, Academia Sinica, Beijing); and Mengxian 
Ding (Changchun Institute of Applied Chemistry, Academia Sinica, Changchun).

\section{Symposium F: Functional Polymer Materials}

This symposium will focus on but not be limited to: conductive polymers, separation membranes, liquid-crystal polymers, shape-memory polymers, damping polymers, piezo-polymers, and photoelectric polymers.

Chairs: Fosong Wang (Academia Sinica, Beijing); Hideki Shirakawa (University of Tsukuba); Tisato Kajiyama (Kyushu University); and Renyuan Qian (Institute of Chemistry, Academia Sinica, Beijing).

\section{Symposium G: Intermetallic Compounds and High Temperature Materials}

Topics will span: intermetallic compounds; high temperature alloys, new high temperature materials, advances in processing and physical metallurgy, mechanical behavior, environmental behavior and coating, and new surface treatment processing.

Chairs: Dongliang Lin (Shanghai Jiao Tong University, Shanghai); C.T. Liu (Oak Ridge National Laboratory, TN); Carl H. Lund (Martin Marietta, IL); Zengyong Zhong (Central Iron \& Steel Research Institute, Beijing); and Guoliang Chen (Beijing University of Science and Technology).

\section{Symposium H: Laser and Particle-Beam Interactions with Solids}

This symposium will discuss laser and ion beam-surface interactions, including both fundamental and applied topics. Areas of interest include but are not limited to:

- Ion implantation;

- Laser and ion beam mixing;

- Laser and ion beam surface treatment;

- Amorphization, metastable phase formation and phase transformations induced by energy beams;

- Laser and ion-beam-assisted deposition;

- Ion beam techniques;

- Beam-induced chemical processes;

- Beam-induced processing;

- Characterization of beam modified microstructures.

Chairs: Shichang Zou (Shanghai Institute of Metallurgy, Academia Sinica, Shanghai); S. Thomas Picraux (Sandia National Laboratories, Albuquerque, NM); Emanuele Rimini (University of Catania, Italy); Zhonglie Wang (Peking University, Beijing); and Baixin Liu (Tsinghua University, Beijing).

\section{Symposium I: Thin Films}

This symposium will cover preparation of thin films and characterization techniques, properties of thin films, including electronics and optics, and metallurgical coatings.

Chairs: Lin Li (Institute of Physics, Academic Sinica, Beijing); Akira Kinbara (University of Tokyo, Tokyo); Avishay Katz (AT\&T Bell Laboratories, Murray Hill, NJ); K. Reichelt (Institute of Film and Ion Technique KFA, Jülich); and Lishi Wen (Institute of Metal Research, Academia Sinica, Shenyang).

\section{Symposium J: Fatigue and Fracture Behavior in Materials}

This symposium is devoted to the fundamentals and applications of fatigue and fracture research in advanced structure materials. The following topics will be explored:

- Fracture aspects in advanced materials, including metallic and polymeric composites, ceramics, etc.;

- Microscopic versus continuum description of fatigue crack propagation, especially in initiation/short crack stage;

- Fatigue criteria and life prediction under spectrum, multi-axial/mixed loading;

- Environmental effect on fatigue and fracture in structural materials;

- High temperature deformation and failure.

Chairs: Minggao Yan (Institute of Aeronautical Materials, Beijing); R.O. Ritchie (University of California, Berkeley); K.J. Miller (Sheffield University, United Kingdom); Zhongguang Wang (Institute of Metal Research, Academia Sinica, Shenyang).

\section{Symposium K: Recent Advances of Tribomaterials}

This symposium involves metals, ceramics, polymers, composite materials, surface coatings, surface modification, solid lubrications, and additives in lubricant, and also aspects of their mechanism studies. Papers are solicited on the following subjects:

- Mechanism and modeling of friction and wear of tribomaterials;

- Friction and wear behavior of new wear resistant materials;

Hard coating;

- Solid lubricants and high temperature lubrication:

- Tribochemistry of surface films;
- Friction and wear behavior of new additives in lubricant.

Chairs: Shizhuo Li (Institute of Metal Research, Academia Sinica, Shenyang); Marshall B. Peterson (Wear Sciences, Maryland); and Jiajun Liu (Tsinghua University, Beijing).

\section{Symposium L: Biomedical Materials}

This symposium will bring together scientists, engineers and clinicians working with all types of biomedical materials. Symposium will span but not be limited to: - Biocompatibility (soft tissue, hard tissue and blood);

- Natural/synthetic biomaterials;

- Clinical application of biomaterials;

- Materials for blood purification;

- Cardiovascular prosthesis;

- Drug delivery systems;

- Materials for biotechnology;

- Dental materials;

- Bioactive ceramic materials;

- Metallic implants.

Chairs: Han Zou (Ji Nan University, Guangzhou); Allan S. Hoffman (University of Washington, Seattle); Yoshito Ikada (Kyoto University, Kyoto); Tingfei Xi (National Institute of the Control for Pharmaceutical and Biological Products, Beijing).

\section{Symposium M: Materials Design}

This symposium will cover prediction of properties of materials through designing of structures at various levels:

- Electronic level calculations;

- Atomic-molecular level calculations;

- Stability of stable and metastable phases;

- Quantitative relationships between properties and structural parameters;

- Environmental effects on structures;

- Artificial microstructure and nanophase materials design;

- Expert systems for materials design.

Chairs: Jimei Xiao (Beijing University of Science and Technology, Beijing); John Radavich (Purdue University, West Lafayette, IN); John D. Dow (University of Notre Dame, IN); Jiajiong Xiong (Tsinghua University, Beijing).

\section{Symposium N: Frontiers of Materials Research}

Chairs: Dongsheng Yan (Academia Sinica, Beijing) and Changxu Shi (National Natural Science Foundation of China, Beijing). 